\title{
Impending compartment syndrome of the forearm and hand after a pressurized infusion in a patient under general anesthesia
} -A case report-

\author{
Chi Yun Sung, Rack Kyung Chung, Yoon Suk Ra, Hee Seung Lee, and Guie Yong Lee \\ Department of Anesthesiology and Pain Medicine, School of Medicine, Ewha Womans University, Seoul, Korea
}

A 74-year-old woman underwent posterior lumbar decompressive fusion at L4-5 for treating spondylolisthesis, with the patient under general anesthesia and she was in the prone position. Following attempts to transfuse blood using a pressurized bag, the intravenous infusion site of the left hand along with the noninvasive blood pressure cuff was changed. Swelling and several bullae on the left forearm and hand were visible. Removal of intravenous catheter, hyaluronidase injection, wet dressing were subsequently performed. In postanesthesia recovery unit, the patient did not complain of pain, and the radial pulse and oxygen saturation of the left appeared normal. Three days after the incident, the edema on the patient's forearm and hand subsided, and the patient was discharged without any complications two weeks afterwards. Impending compartment syndrome should be given close attention, and particularly when performing pressurized infusion in patients who are unable to express pain because they are under general anesthesia. (Korean J Anesthesiol 2011; 60: 60-63)

Key Words: Compartment syndrome, General anesthesia, Infusion, Pressure.

Compartment syndrome is a potentially devastating complication that can occur during the perioperative period. The etiological agents vary, and they include multiple closed fractures, soft tissue and after-arterial injuries, burns and the intravenous injection of hypertonic solution or vasoactive drugs
[1]. Nevertheless, the incidence of compartment syndrome due to a pressurized infusion is rare [2]. We report here on a case of impending compartment syndrome that was the result of a pressurized infusion in a patient who was under general anesthesia and in the prone position.

Received: June 1, 2010. Revised: 1st, June 18, 2010; 2nd, September 13, 2010. Accepted: September 29, 2010.

Corresponding author: Rack Kyung Chung, M.D., Department of Anesthesiology and Pain Medicine, School of Medicine, Ewha Womans University, 911, Mok 5-dong, Yangcheon-gu, Seoul 158-710, Korea. Tel: 82-2-2650-5039, Fax: 82-2-2655-2924, E-mail: rkchung@ewha.ac.kr The 86th Annual Scientific Meeting of the Korean Society of Anesthesiologists, 2009, Jeju Convention Center.

(c) This is an open-access article distributed under the terms of the Creative Commons Attribution Non-Commercial License (http:// creativecommons.org/licenses/by-nc/3.0/), which permits unrestricted non-commercial use, distribution, and reproduction in any medium, provided the original work is properly cited. 


\section{Case Report}

A 74-year-old woman (body weight: $60 \mathrm{~kg}$, height: $149 \mathrm{~cm}$, ASA physical status: 2) was scheduled for posterior lumbar decompressive fusion surgery at L4-5 under the diagnosis of spondylolisthesis. The patient's previous medical history entailed ten years of diabetes, which was well-controlled with oral hypoglycemic agents. Two weeks prior to the operation, she had undergone cervical intervertebral anterior fusion at C7-T1 and she had also been taking methylprednisolone 750$1,000 \mathrm{mg}$ daily for 11 days. The patient was placed under general anesthesia via a preexisting 18-gauge IV catheter on the right forearm with $0.9 \%$ normal saline. A noninvasive blood pressure (NIBP) cuff and an additional 18-gauge IV catheter with $0.9 \%$ normal saline were applied through the cephalic vein at the level of the left forearm. After performing Allen's test, a radial artery cannulator was inserted at the right side and then the NIBP on the left arm was checked every 30 minutes. General anesthesia was induced with thiopental sodium, midazolam and fentanyl and it was maintained with sevoflurane- $\mathrm{N}_{2} \mathrm{O}$ in oxygen after tracheal intubation. The patient's position was changed to prone using a Wilson's table with her arms extended at the shoulders and the elbows naturally flexed. She was covered with a warming blanket (Bair hugger ${ }^{\circledR}$, Augustine medical Inc, Eden Prairie, MN, USA) over both arms and the upper body. Both IV routes were confirmed to be functioning well. Normal saline $550 \mathrm{ml}$ was infused thorough the left IV catheter by gravity for the first 3 hours of the operation. As the blood loss increased, an infusion of blood under pressure was applied. Approximately 20 minutes after the pressurized infusion on left forearm, the anesthetist found problems of

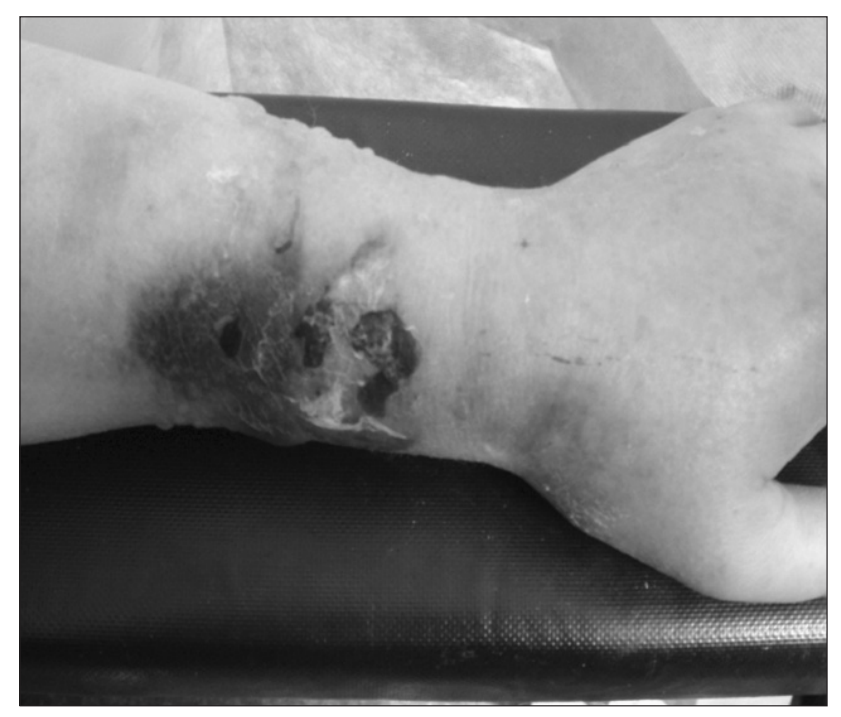

Fig. 1. Left forearm with extensive edema, multiple bullae and bruise. dripping of the left IV fluid. To confirm the IV site, the warm blanket was uncovered, and several $2-3 \mathrm{~cm}$ - sized bullae, a big bruise and tensely pale swelling from the palm to the upper part of the forearm were discovered on the confirmed left IV site (Fig. 1). During this period, a total of $550 \mathrm{ml}$ of normal saline that was infused by gravity and $50 \mathrm{ml}$ of packed red blood cells and $150 \mathrm{ml}$ of normal saline via a pressure bag inflated to $200 \mathrm{mmHg}$ had been injected through a cephalic vein of the left forearm. The process of refilling the digital capillaries took over 4 seconds, and arterial pulsation was weakly palpable. The NIBP cuff and the left forearm IV catheter were removed under the presumptive diagnosis of impending compartment syndrome. A plastic surgeon was quickly consulted and we injected hyaluronidase $1,500 \mathrm{U}\left(\mathrm{H}_{-} \mathrm{LASE}^{\circledR}, \mathrm{L} \& \mathrm{H}\right.$ Pharm, Korea) subcutaneously on the patient's forearm and hand to decompress the increased pressure of the compartments by absorbing the extravasated blood and fluid (Fig. 2). The plastic surgeon removed the multiple bullae and placed a wet dressing. The patient's arms were elevated above the heart level while the operation was continued. After 4 hours of operation, the patient was extubated in the operating room and then moved to the recovery room. In the postanesthesia recovery unit, a physical examination revealed no signs of motor weakness, sensory abnormality or color change on the left forearm and hand. The radial arterial pulsation and capillary filling had recovered and the edema was slightly decreased. The pulse oximeter showed $100 \%$. As the patient did not complain of pain on passive movement, she was transferred to the general ward. The edema on the left forearm and hand subsided in three days and the patient was discharged without any complications about her left forearm two weeks later.

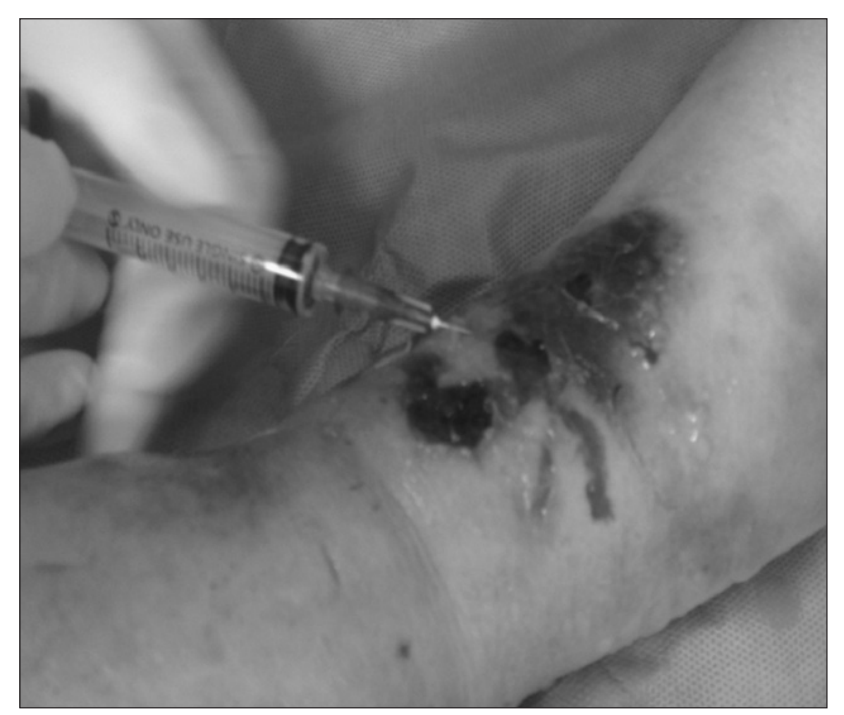

Fig. 2. Subcutaneous injection of hyaluronidase into the forearm. 


\section{Discussion}

Compartment syndrome defined as a condition in which the circulation and function of the tissue within a closed space are compromised by increased pressure inside that space [3]. Cases of compartment syndrome on the upper arm have not been frequently reported. The common causes of compartment syndrome are muscle avulsion, bone fracture of the tibia or forearm, and external compression [1]. Iatrogenic causes by extravasation related to anesthesia have been reported with drugs like diazepam and antibiotics [4], hyperosmolar solution such as mannitol [5], hypertonic saline [6], a pressurized IV infiltration [2], Bier block [7] and arterial cannulation [8].

The pathophysiology of compartment syndrome is caused by increased intracompartmental pressure that is strong enough to impede microcirculatory perfusion, and this results in ischemic injury to muscle, nerves and other compressed structures. Intravenous infiltrations have been classified into 4 stages and the first is local pain without swelling or redness, the second is pain, redness and minimal swelling, the third is moderate swelling and blanching, and the forth is capillary refilling of more than 4 seconds and skin breakdown [9].

Matsen [3] found that neural abnormality began after 30 minutes of ischemia and this became irreversible after 12 hours, the permeability of the capillary endothelium increased after about 3 hours of ischemia, and muscle changes began after about 2 hours and this became irreversible after 4 hours of ischemia.

The importance of making an early diagnosis and treatment involving hyaluronidase and fasciotomy cannot be overemphasized. A failure to diagnose impending compartment syndrome by the time of emergence from anesthesia may result in permanent injury.

The diagnosis of compartment syndrome is based on intracompartmental pressure measurement by a wick catheter, simple needle manometry, an infusion technique and using a slit catheter. The definitive diagnosis is made after directly measuring the intracompartmental pressure. The threshold of the compartment pressure that requires treatment is controversial, but the indications for fasciotomy includes the clinical signs, symptoms and an intracompartmental pressure of 15-25 mmHg, or in the absence of clinical sign andsymptoms, an intracompartmental pressure of more than $25 \mathrm{mmHg}$ [1]. However Tobias et al. [10] treated compartment syndrome conservatively without fasciotomy in spite that the interstitial pressure of the biceps brachii and triceps brachii muscle was over $30 \mathrm{mmHg}$.

It is difficult to diagnose compartment syndrome early in the case of an anesthetized or obtunded patient who is incapable of expressing pain [9]. Therefore, frequent examination of the extremities is crucial to make a timely diagnosis and minimize the risk of permanent injury. Monitoring includes inspection, pulse oximetry and assessing the intraarterial pressure wave of the upper arm. This is critical in obtunded or anesthetized patients [11].

Once the injury occurs, the methods of management can be either operative or non-operative. Non-operative management involves daily cleansing and topical application of antiseptic cream, enzymatic debridement, hyaluronidase application, subcutaneous saline flush-outs and liposuction [12]. Laurie et al. [13] used topical hyaluronidase in an extravasation injury of a rabbit model. They found it effective in reducing the skin necrosis caused by extravasated toxins. Heclker [14] applied hyaluronidase less than an hour after extravasation, and none of the patients suffered full-thickness skin defects or weakness of the motion of any joint. A definite surgical treatment for severe compartment syndrome is fasciotomy. Fasciotomy decompresses the pressure of a compartment and prevents permanent injury of nerves and vessels. Spenny et al. [4] and Tiwari et al. [9] reported that their patients recovered from compartment syndrome without complication by performing fasciotomy. The wait-and-see approach is strongly contraindicated for treatment, and this approach can quickly result in permanent sequelae.

The effectiveness and significance of prevention cannot be emphasized enough. Khan and Holmes [15] established the protocol and kit to prevent extravasation, which consists of large central vein catheterization, avoidance of multiple perforations on same the vein, avoidance of perfusion under pressure, close monitoring of the venous puncture site, avoidance of sites on hands and near joints and avoidance of limbs with impaired circulation.

In our case, at first the skin lesion such as bullae could be considered to be caused by the heat of the warming blanket. But the warming blanket covering the upper body and both arms did not contact directly with in skin and it did not increase the temperature enough to cause the bullae. With the abrupt onset and no allergy history, the signs also excluded the possibility of necrotizing fasciitis or cellulitis. In this case, we considered impending compartment syndrome that was due to the extravasation from a pressurized infusion of normal saline and packed red blood cells, although we did not measure the intracompartmental pressure.

There were several factors that adversely affected the local tissue blood flow and perfusion to produce compartment syndrome on the forearm. The patient's skin and vessels were thin and they lacked elasticity due to her diabetes and the preoperative steroid medication. Moreover, we performed a pressurized intravenous infusion and put the blood pressure cuff on the same side and we repeatedly examined the blood 
pressure every 30 minutes. Extravasation of the fluids may have increased the pressure within a limited space and worsened the circulation below the blood pressure cuff. As the thin walls of the venules were pressured and might be ruptured, the administrated fluid and blood were extravasated and this increased the capillary perfusion pressure and caused compartment pressure and high hydrostatic pressure, which all ultimately resulted in edema and bullae [4]. There have been a few cases of compartment syndrome from pressurized infusion like our report $[2,10]$. Another adverse factor may be the patient's age, which increases the vulnerability of skin and also vein fragility [4].

In this reported case, treatment was given rapidly and noninvasively by the consulted plastic surgeon. Subcutaneously or intramuscularly injected hyaluronidase absorbs body fluid or blood components in connective tissue, it lowers the compartmental pressure and it prevents the injury of nerves and vessels.

In conclusion, compartment syndrome is a rare complication of extravasation injury due to pressurized infusion. It can increase the possibility of permanent injury in nerves and vessels if it is not treated in a timely manner. Anesthesiologists have to be even more attentive to closely monitor all intravenous sites, and particularly because a patient is unable to complain of their pain when under general anesthesia, or a patient has more vulnerable veins due to use of steroid or the difficulty of venous access.

\section{References}

1. Ouellette EA, Kelly R. Compartment syndromes of the hand. J Bone Joint Surg Am 1996; 78: 1515-22.

2. Willsey DB, Peterfreund RA. Compartment syndrome of the upper arm after pressurized infiltration of intravenous fluid. J Clin Anesth 1997; 9: 428-30.

3. Matsen FA 3rd. Compartmental syndrome. An unified concept. Clin Orthop Relat Res 1975; (113): 8-14.

4. Spenny ML, Moen KY, Dinulos JG. Acute bullous eruption with compartment syndrome due to intravenous infiltration. Arch Dermatol 2004; 140: 798-800.

5. Edwards JJ, Samuels D, Fu ES. Forearm compartment syndrome from intravenous mannitol extravasation during general anesthesia. Anesth Analg 2003; 96: 245-6.

6. Quigley JT, Popich GA, Lanz UB. Compartment syndrome of the forearm and hand: a case report. Clin Orthop Relat Res 1981; (161): 247-51.

7. Ananthanarayan C, Castro C, McKee N, Sakotic G. Compartment syndrome following intravenous regional anesthesia. Can J Anaesth 2000; 47: 1094-8.

8. Qvist J, Peterfreund RA, Perlmutter GS. Transient compartment syndrome of the forearm after attempted radial artery cannulation. Anesth Analg 1996; 83: 183-5.

9. Tiwari A, Haq AI, Myint F, Hamilton G. Acute compartment syndromes. Br J Surg 2002; 89: 397-412.

10. Tobias MD, Hanson CW 3rd, Heppenstall RB, Aukburg SJ. Compartment syndrome after pressurized infusion. Br J Anaesth 1991; 67: 332-4.

11. Ouellette EA. Compartment syndromes in obtunded patients. Hand Clin 1998; 14: 431-50.

12. Kumar RJ, Pegg SP, Kimble RM. Management of extravasation injuries. ANZ J Surg 2001; 71: 285-9.

13. Laurie SW, Wilson KL, Kernahan DA, Bauer BS, Vistnes LM. Intravenous extravasation injuries: the effectiveness of hyaluronidase in their treatment. Ann Plast Surg 1984; 13: 191-4.

14. Heckler FR. Current thoughts on extravasation injuries. Clin Plast Surg 1989; 16: 557-63.

15. Khan MS, Holmes JD. Reducing the morbidity from extravasation injuries. Ann Plast Surg 2002; 48: 628-32. 